Sustainability researchers will need to follow a multidisciplinary - nay, transdisciplinary - approach that goes beyond what many scientists have been used to. Future Earth's 'co-design' intends natural and social scientists to plan and carry out research with outside experts. Whether that will win over academic researchers, stakeholders and, crucially, funders remains to be seen. To convince sceptics, the scheme needs to provide a successful example of how it will work in practice.

Preservation of the natural commons, such as atmosphere, water, land and oceans, for future generations is vital and a cause to which any responsible scientist will happily subscribe. But combining the conventional scientific methods of the natural and social sciences with knowledge from various other sources - land owners and planners, insurance companies, conservation groups, emergency organizations and political decision-makers - poses conceptual and organizational challenges.

A cross-community Future Earth workshop on adaptation and responses to extreme climate events, held last month in Berlin, offered a taste of such challenges (see go.nature.com/6utfmi) and might serve as a test for the design of research networks on sustainability issues. Under time pressure, participants had to draft a research strategy to address the drivers and implications of extreme events, and make it fit with Future Earth's conceptual framework - a tough issue. The workshop asked scientists from different academic cultures to do that work, which produced semantic confusion and the odd unhelpful generalization.

But the workshop was not in vain. Many participants (a healthy number of whom were from developing nations) said that they revelled in being pushed out of their comfort zones. They produced several meaty research questions, including some genuinely new ideas for how the social and natural sciences could interact. For example, when do climatic and socio-economic factors combine to amplify the impacts of climate extremes and induce cascading harm? Are there 'tipping points' at which social or natural systems might fail to recover from shocks? And how might science-based adaptation work in data-scarce regions?

The ideas found an audience. Representatives of funding agencies at the workshop cautiously indicated that the proposals stand a good chance of getting funded by the Belmont Forum, a worldwide group of 21 major funders of global environmental-change research.

But governments and grant-giving agencies have not yet firmly committed to funding Future Earth as a whole. The reluctance comes from uncertainty over what the scheme might be able to deliver. The closure of successful programmes in favour of something fashionable but conceptually unproven has earned Future Earth sceptical glances.

"Sustainability
research must
not become
tied in the
straitjacket of
conceptualism."
But then, it was launched in response to complaints that previous programmes were not sufficiently linked and that the knowledge they produced was scarcely picked up in practice. There is no lack of studies, for example, on how extreme heat, rain and wind affect farmers, city dwellers and coastlines in many parts of the world. But the results are almost useless if they never make it beyond the pages of academic journals.

Future Earth will need to make sure that scientific evidence comes to the desks of decision-makers, no matter what they might then make of it. But the programme should also avoid retreading familiar ground. The mountain of data from previous programmes, including countless climate-change studies, remains relevant - even if the information hasn't yet been put to constructive use.

Future sustainability research, no matter how interdisciplinary, should build on that heritage and focus on finding and closing knowledge gaps. In doing so, scientists involved in Future Earth can provide an invaluable service to society. And researchers in niche disciplines - palaeoclimatology or behavioural science, say - who work to fill those gaps will get a welcome chance to put their work into a broader context.

Future Earth might also become a showcase for linking natural and social sciences - a real necessity given that human activity is altering the planet at worrying speed. But sustainability research must not become tied in the straitjacket of conceptualism and utilitarianism. Scientists are not merely service providers. As in any other field of science, sustainability research must remain at its core a curiosity-driven affair.

\section{Brain power}

\section{As brain stimulation finds non-medical uses, now is the time to consider its implications.}

$\mathrm{I}$ $\mathrm{t}$ is a cautionary tale for twenty-first-century medicine. Late last year, neurosurgeons in the United States reported odd symptoms in three of their patients. Well into their sixties and seventies, these people complained of headaches, nausea, unstable balance, weak legs, low blood pressure and falling down. Chest X-rays revealed the problem. Two devices implanted into their chests - a pacemaker to help their failing hearts and a battery unit that powered electrodes buried deep inside their heads to control the signature tremors of Parkinson's disease - had been placed too close together. One machine was interfering with the functioning of the other (M. Sharma et al. Basal Ganglia 6, 19-22; 2016).

From iron lungs and dialysis machines to implantable defibrillators, we are used to technology helping our bodies. Deep-brain stimulation - the electrodes and battery implanted in the patients' heads - has been helping people with neurological and psychiatric disorders for more than a decade, but it requires quite a commitment. Brain surgery is expensive and not for everybody. The number of people who might benefit is very small given the overall burden posed by mental illness and related problems. This is one reason why there is a lot of interest in cheaper and easier types of brain stimulation, which apply electric current and magnetic fields to the outside of the head.

If these types of brain stimulation are found not to produce much of a difference, then it will not have been for a lack of effort. Academic journals are filling up with case reports and preliminary trials of the technologies to help people with depression, autism spectrum disorders, schizophrenia, obsessive-compulsive disorder, addiction, anxiety and many more cognitive problems. It is early days, but there are enough positive results to draw the attention of people who struggle with such issues or know someone who does. Some of these people want to try it on themselves or their children. An electrical brain stimulator is fairly simple to make, and even simpler to buy from one of the companies that are popping up to sell them online. Self-medication has never been so high-tech.

Many neuroscientists have raised the alarm over do-it-yourself(DIY) brain stimulation, pointing out that it can be unsafe in the short term and might have side effects in the long term. Some want regulation. But there is another, more fundamental, ethical issue that must be confronted. As we report in an Outlook article on page S6 - one of a series of pieces that discuss cognitive enhancement - the use of DIY brain stimulators is not confined to those for whom conventional medicine has failed. A small but growing number of people want to use the devices to improve their natural mental abilities. And in so doing, this community is piggy-backing on scientific studies that suggest that electric currents and magnetic fields could improve academic performance by boosting memory and attention, and perhaps even alter attitudes.

The use of medicines to enhance performance in sport is frowned on, and a clear line has been drawn between taking them to treat and taking them to cheat. Could a similar distinction be made for cognitive- $\rightarrow$ NATURE.COM To comment online, click on Editorials at: go.nature.com/xhunqv enhancement techniques? Should it be? It's too soon to answer some of these questions - scientists and doctors must first reach consensus on the effectiveness of the techniques - but it is not too soon to ask them. 